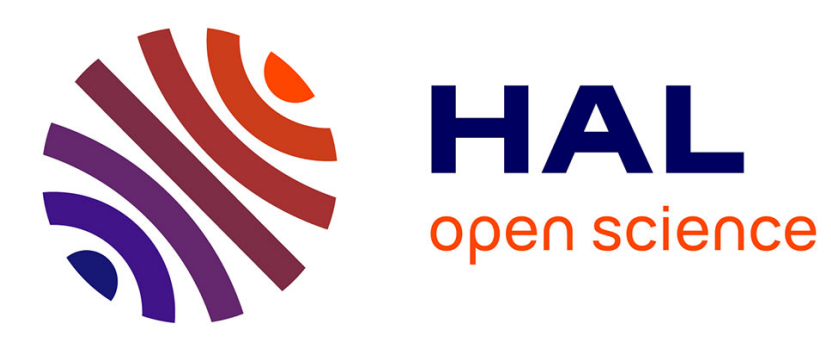

\title{
Influence of the Mass ratio on the fluid-elastic instability of a flexible cylinder in a bundle of rigid tubes
}

\author{
Gilles Harran
}

\section{To cite this version:}

Gilles Harran. Influence of the Mass ratio on the fluid-elastic instability of a flexible cylinder in a bundle of rigid tubes. Journal of Fluids and Structures, 2014, vol. 47, pp. 71-85. 10.1016/j.jfluidstructs.2013.11.005 . hal-01068986

\section{HAL Id: hal-01068986 \\ https://hal.science/hal-01068986}

Submitted on 26 Sep 2014

HAL is a multi-disciplinary open access archive for the deposit and dissemination of scientific research documents, whether they are published or not. The documents may come from teaching and research institutions in France or abroad, or from public or private research centers.
L'archive ouverte pluridisciplinaire HAL, est destinée au dépôt et à la diffusion de documents scientifiques de niveau recherche, publiés ou non, émanant des établissements d'enseignement et de recherche français ou étrangers, des laboratoires publics ou privés. 


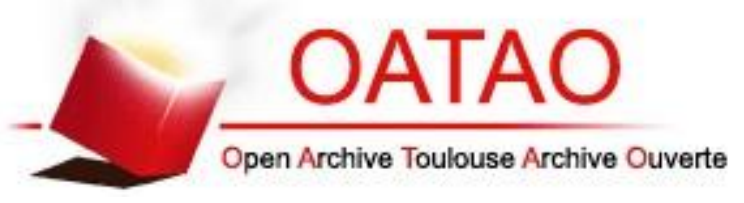

Open Archive Toulouse Archive Ouverte (OATAO)

OATAO is an open access repository that collects the work of Toulouse researchers and makes it freely available over the web where possible.

This is an author-deposited version published in: http://oatao.univ-toulouse.fr/ Eprints ID: 11252

Identification number: DOI : 10.1016/j.jfluidstructs.2013.11.005

Official URL: http://dx.doi.org/10.1016/j.jfluidstructs.2013.11.005

\section{To cite this version:}

Harran, Gilles Influence of the Mass ratio on the fluid-elastic instability of a flexible cylinder in a bundle of rigid tubes. (2014) Journal of Fluids and Structures, vol. 47 . pp. 71-85. ISSN 0889-9746

Any correspondence concerning this service should be sent to the repository administrator: staff-oatao@inp-toulouse.fr 


\title{
Influence of the mass ratio on the fluidelastic instability of a flexible cylinder in a bundle of rigid tubes
}

\author{
G. Harran * \\ Institut de Mécanique des Fluides de Toulouse, 6 allée du Prof. C. Soula, 31400 Toulouse, France
}

\begin{abstract}
A B S T R A C T
Several linear lumped-parameter models were proposed in the past to identify the main mechanisms underlying the cross-flow instability of a single flexible cylinder in tube bundles. Basing on such models, we analyze the influence of the mass ratio when the cylinder vibrates in the transverse direction, without structural damping (corresponding to a zero Scruton number). For two selected mass ratios, we focus on this linear interaction plotting the poles of the fluid-structure system as a function of the reduced velocity (root locus). This asymptotic approach allows a better understanding of the combined influence of the transient fluidelastic coupling and the mass ratio.
\end{abstract}

Keywords:

Fluidelastic instability

Tube bundles

Cross-flow

Root locus

\section{Introduction}

The fluidelastic interaction in cylinder arrays is complex, since it combines three types of instability (Khalifa et al., 2012; Païdoussis et al., 2010; Blevins, 1994):

- Instability by antisymmetric stiffness similar to classical flutter, with at least two degrees of freedom involved in the motion. It is the main mechanism when reduced velocity is much higher than unity.

- Dynamic instability for a Single-Degree-Of-Freedom cylinder. It is the main mechanism when reduced velocity is of the order of unity.

- Static instability (divergence) for a Single-Degree-Of-Freedom cylinder. It was highlighted by Paidoussis et al. (1989) for peculiar geometrical patterns.

The difficulty to differentiate these instabilities can be evoked as one of the reason for the high experimental scattering on the values of critical velocity $U_{c}$ or the reduced critical velocity

$$
U_{r c}=\frac{U_{c}}{f_{S} D}
$$

where $f_{S}$ is the structural natural frequency and $D$ the cylinder diameter. In addition to the geometric pattern influence, $U_{c}$ is affected by other phenomena:

- Vortex-Induced-Vibration domain and Movement-Induced-Vibration domain overlap when Scruton number is low (Weaver, 2008). According to Granger and Paidoussis (1996), this can explain some modeling difficulties for fluidelastic instability prediction.

\footnotetext{
* Tel.: + 33534322884 .

E-mail addresses: Gilles.Harran@imft.fr, harran@imft.fr
} 
Table 1

Nominal parameters for the experiment and simulations.

\begin{tabular}{lll}
\hline Parameter & Notation & Value \\
\hline Fluid density $\left(\mathrm{kg} \mathrm{m}^{-3}\right)$ & $\rho_{F}$ & 1.2 \\
Dynamic viscosity $\left(\mathrm{m}^{2} \mathrm{~s}^{-1}\right)$ & $\nu$ & $15 \times 10^{-6}$ \\
Cylinder length $(\mathrm{m})$ & $L$ & 0.6 \\
Cylinder diameter $(\mathrm{m})$ & $D$ & 0.08 \\
Pitch (m) & $P$ & 0.12 \\
Pitch ratio & $P / D$ & 1.5 \\
Aspect ratio & $a=(P / D) /(P / D-1)$ & 3 \\
Total mass (kg) & $M_{S}$ & 0.5 \\
Mass per length (kg/m) & $m=M_{S} / L$ & 0.83 \\
Structural damping coefficient $\left(\mathrm{N} /\left(\mathrm{ms}^{-1}\right)\right)$ & $C_{S}$ & 0 \\
Structural stiffness coefficient $\left.(\mathrm{N} \mathrm{m})^{-1}\right)$ & $K_{S}$ & 3000 \\
Structural reduced damping & $\xi_{S}$ & 0 \\
Structural natural frequency $(\mathrm{Hz})$ & $f_{S}$ & 12.3 \\
Structural natural frequency $(\mathrm{rd} / \mathrm{s})$ & $\omega_{S}$ & 77.46 \\
Pitch Reynolds number & $\operatorname{Re}=U_{p} D / \nu$ & 51500 \\
Mass ratio & $m^{\star}=m /\left(\rho_{F} D^{2}\right)$ & 108 \\
Scruton number & $\mathrm{Sc}=2 \pi \xi_{S} m^{\star}$ & 0 \\
Reduced velocity & $U_{r}=U /\left(f_{S} D\right)$ & 3.26 \\
\hline
\end{tabular}

- The Reynolds number strongly influences the physics of the flow, but it is very seldom taken into account. Gillen and Meskell (2008) show an important influence on the static efforts and notable consequences on the critical velocity by the quasi-unsteady model of Granger and Paidoussis (1996).

This critical velocity value is a crucial criterion for design of heat exchangers. It is usually plotted as a function of the mass ratio $\left(m^{\star}\right)$ and reduced structural damping $\left(\xi_{S}\right)$, defined in Table 1 . The three constants $K, p, q$ in relation (1)

$$
U_{r c}=K\left(m^{\star}\right)^{p} \xi_{S}^{q}
$$

are then fitted from experimental data. The Scruton number $\left(\mathrm{Sc}=2 \pi \xi_{S} m^{\star}\right)$ appears if $p=q$ is considered. This dimensionless group quantifies the ratio between the energy dissipated by the structure and the energy taken from the fluid (Axisa, 2001). Since experimental evidence of a specific dependence to the structural damping and the mass ratio are numerous (Tanaka and Takahara, 1981; Price, 2001), it is not relevant to assume that $p=q$, as suggested by Connors-type equations (Connors, 1970).

Some efforts are still necessary to unfold the specific influence of the mass ratio and reduced structural damping according to the value range of the Scruton number (Weaver, 2008). In order to contribute to this analysis, we propose to isolate the influence of the mass ratio, considering an undamped structure, which corresponds to the asymptotic case $S c \rightarrow 0$. The linear models with lumped-parameter approach will be considered, leading to a user-friendly description of the fluidelastic instability in cross-flow cylinder arrays. Nevertheless, they show substantial weakness in accurately predicting critical velocities for specific industrial applications such as steam generators (Price, 2001). This industrial application is characterized by low Scruton numbers and reduced velocities of the order of unity. That implies a strong fluidelastic coupling and the need for taking into account of the transients of the coupling mechanism (Tanaka and Takahara, 1981). For a bibliographical synthesis on these phenomena and the various modeling options to predict these instabilities (generally referred as damping-controlled instability), one can consult (Price, 2001; Païdoussis et al., 2010).

We propose to distinguish two classes of models, according to whether the fluid-structure system is described by:

- a model of the same order as that of the structure model order. The interaction is then characterized by a damping coefficient and a stiffness coefficient added by the flow, which depend on the reduced velocity, since the quasi-static theory is not valid. It is for example the approach of Chen (1987).

- a model of a higher order than that of the structure model order. The interaction is identified by its own dynamics. It is for example the approach of Price and Païdoussis (1986) or Granger and Paidoussis (1996), that we will compare (Section 2.3). The model of Lever and Weaver (1982) also belongs to this category.

In order to analyze the influence of the mass ratio on the loss of stability, it is necessary to precisely describe the dynamics of the interaction and not only its consequences on the dominant mode of the coupled system. Only the second category of models can thus bring elements of physical insight. This is why we will limit the discussion to this class of models. Only linear models are considered here, but the nonlinear tools for simulation are set up for the continuation of the study. We will use the symbolic formalism for its ease of handling, although the temporal formulation by the operator of convolution is more explicit in describing the physics of the memory.

All the cylinders are fixed, except the central one that is flexibly mounted. It can move in the $z$ direction only in an in-line square array, with $P$ the pitch between cylinders (Fig. 1). The ratio between the pitch velocity $U_{p}$ and the free-stream single 


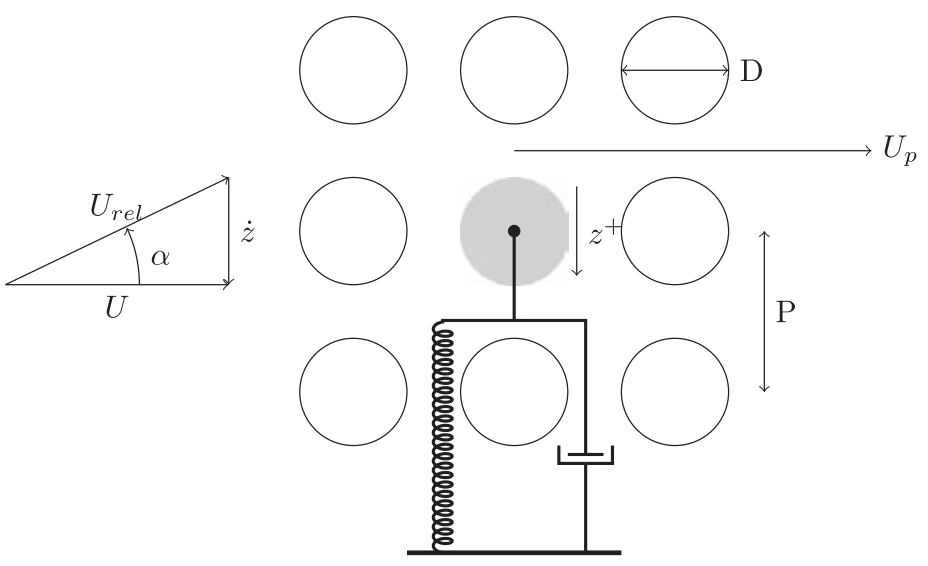

Fig. 1. Flexibly mounted cylinder in an in-line square array.

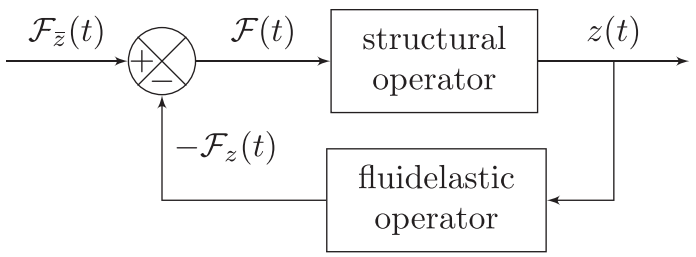

Fig. 2. Functional diagram of the fluidelastic closed-loop system.

phase flow velocity $U$ is the aspect ratio $a=U_{p} / U=(P / D) /(P / D-1)$. The numerical values of the simulated configuration and the notations are given in Table 1 . It corresponds to an experimental setup under development in a wind tunnel, with virtualization of the dynamics of structure as proposed by Hover et al. (1998) and later by Mackowski and Williamson (2011). This concept will allow the experimental study of the asymptotic case of an undamped structure $\left(\xi_{S}=0\right)$, analyzed in this paper.

\section{Fluidelastic models}

After a short presentation of the interaction concept with a block diagram representation (Preumont, 2002), the fluidelastic coupling models based on the lumped-parameter approach are recalled and discussed.

\subsection{Block-diagram representation}

Following a common assumption (Chen and Jendrzejczyk, 1983), the fluid loading on the structure $\mathcal{F}$ can be separated into two terms: a force non-correlated to the cylinder movement $\mathcal{F}_{\bar{z}}(t)$ and a force correlated to the cylinder movement $\mathcal{F}_{z}(t)$, leading to

$$
\mathcal{F}(t)=\mathcal{F}_{\bar{z}}(t)+\mathcal{F}_{z}(t) .
$$

The feedback nature of fluidelastic systems is considered by a functional diagram representation (Fig. 2). This concept allows to characterize the stability of the closed-loop system with the control theory, as emphasized by Fung (1955).

The fluidelastic operator models the feedback of the structure movement on the fluid loading. The structural operator represents the dynamics of the structure. There are differences in the behavior of the structure in vacuum and in quiescent fluid. These differences are important when the mass ratio is low and they are generally taken into account by an added mass and possibly an added damping. In this paper, the term structure refers to the structural behavior in quiescent fluid. So, added mass and added damping are included into the structure parameters.

As a first step, it is often considered that the excitation term of the feedback system $\mathcal{F}_{\bar{z}}(t)$ represents turbulence-induced forces (random and deterministic). But on the one hand, the wake-oscillator concept can be used alternatively to model organized eddies (de Langre, 2006) by a coupling with the structure-oscillator. Lock-in is then interpreted as a classicalflutter instability. This linear model gives a description of the interaction between the structure movement and organizededdies. On the other hand, the organized eddies impact the random turbulence properties: the Von Kármán vortices cause an imbalance between production an dissipation of the turbulent energy cascade, which changes the slope of the power spectral density in the inertial domain, as demonstrated by Braza et al. (2006). So, which part of turbulence has to be interpreted as the excitation loading for the closed-loop system? Aware of the difficulty to clearly define the frontier of the system, we will analyze the stability problem for the Movement-Induced-Vibrations without Turbulence-InducedVibrations or Vortex-Induced-Vibrations with the functional diagram of Fig. 2. We choose a Linear Time Invariant 
representation for both structural and fluidelastic operators. This assumption restricts the model validity around an equilibrium point considering small variations for the state of the system. The equation of the dynamics represented in Fig. 2 can be expressed as

$$
M_{S} \ddot{z}+C_{S} \dot{z}+K_{S} z=\mathcal{F}_{\bar{z}}(t)+\mathcal{F}_{z}(t)
$$

In the Laplace domain this leads to the structural transfer function connecting the position variations and the global fluid loading $\mathcal{F}(t)$

$$
\frac{z(p)}{\mathcal{F}(p)}=\frac{1}{K_{S}+C_{S} p+M_{S} p^{2}}
$$

which is coupled with the fluidelastic transfer function $-\mathcal{F}_{z}(p) / z(p)$, leading to the closed-loop transfer function $z(p) / \mathcal{F}_{\bar{z}}(p)$. Notations are the same in both temporal and Laplace domains and $p=x+j y$ is the symbolic variable.

\subsection{Fluidelastic transfer function}

Generally, the lumped-parameter models for fluidelastic coupling define static parameters and dynamical parameters. Lever and Weaver (1986) describe flow structure (the wake region and the channel region) with static parameters and determine the global load by integrating the pressure over the attached region. The static part of coupling can also result from the drag coefficient $C_{D}$ and the lift coefficient $C_{L}$ as functions of the cylinder position $z$, on which they necessarily depend because of the presence of the adjacent tubes (Price and Païdoussis, 1986; Granger and Paidoussis, 1996). By taking the convention of positive displacement downwards (Fig. 1), Eq. (4) is the expression of the force correlated with the movement

$$
\mathcal{F}_{z}(t)=-T \sin \alpha-P \cos \alpha=-\frac{1}{2} \rho_{F} U_{r e l}^{2} D L\left[C_{D}(z, \dot{z}) \sin \alpha+C_{L}(z, \dot{z}) \cos \alpha\right] .
$$

The linearization of the force coefficients (Price and Païdoussis, 1986) leads to

$$
C_{D}=C_{D_{0}}+\left.\frac{\partial C_{D}}{\partial z}\right|_{0} z=C_{D_{0}}
$$

and

$$
C_{L}=C_{L_{0}}+\left.\frac{\partial C_{L}}{\partial z}\right|_{0} z=\left.\frac{\partial C_{L}}{\partial z}\right|_{0} z
$$

since at the equilibrium point $C_{L_{0}}=\partial C_{D} /\left.\partial z\right|_{0}=0$, for symmetry reason. The subscript 0 corresponds to static values for the equilibrium point.

With the linear assumptions (see Fig. 1) $\alpha=\dot{z} / U$ and $U_{r e l}=\sqrt{\dot{z}^{2}}+U^{2} \approx U$, the force model can be written as

$$
\mathcal{F}_{z}(z, \dot{z}, t)=-\frac{1}{2} \rho_{F} U^{2} D L\left[C_{D_{0}} \frac{\dot{z}}{U}+\left.\frac{\partial C_{L}}{\partial z}\right|_{0} z\right]=-C_{a 0} \dot{z}-K_{a 0} z
$$

with

$$
\begin{aligned}
C_{a 0} & =\frac{1}{2} \rho_{F} U D L C_{D_{0}}, \\
K_{a_{0}} & =\left.\frac{1}{2} \rho_{F} U^{2} L \frac{\partial C_{L}}{\partial Z^{\star}}\right|_{0}, \\
z^{\star} & =\frac{z}{D} .
\end{aligned}
$$

This formulation (5) reflects two effects

- An added damping $\left(C_{a 0}\right)$ function of the drag. This is the stabilizing effect of the drag (as in galloping instability),

- An added stiffness $\left(K_{a 0}\right)$ function of the lift variations with the cylinder position. Measurements show that $K_{a 0}>0$, in the vast majority of tube bundle patterns.

For most geometric patterns, a dynamic instability occurs when reduced velocity is of the order of unity, whereas the quasi-static model (5) predicts unconditional stability. This model must be modified to take into account of the transient effects. In the quasi-unsteady model, Granger and Paidoussis (1996) propose to convolve $z(t)$ the tube displacement into Eq. (5) with $h(t)$ the impulse response of a linear filter. Eq. (6) models the transient process of the lift generating.

$$
\mathcal{F}_{z}(z, \dot{z}, t)=-\frac{1}{2} \rho_{F} U^{2} D L\left[C_{D_{0}} \frac{\dot{z}}{U}+\left.\frac{\partial C_{L}}{\partial z}\right|_{0} z \star h(t)\right] .
$$

In the Laplace domain, the impulse response of the filter $h(t)$ becomes the transfer function $H(p)=\mathcal{L}[h(t)]$ for zero initial conditions, as illustrated in Fig. 3. 


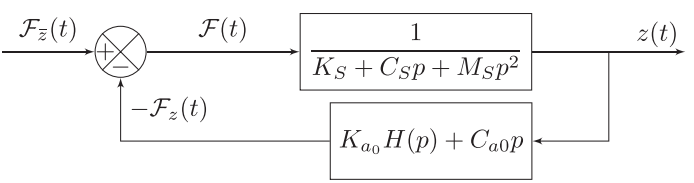

Fig. 3. Functional diagram for SDOF tube when $U_{r} \approx 1$.

If the need for introducing a phase delay to explain instabilities of tube bundles is relatively recent, the aerodynamic community has considered the importance of transients in flutter models approximately sixty years before, with the works of Wagner (1925). Nowadays, modeling is extended to the nonlinear analysis considering the Volterra series or High Order Spectral Analysis (Silva, 2005).

A distinction can be drawn between coupling models depending on whether the process is assumed with memory effects or memoryless:

- memoryless. This is the time delay model (Price and Païdoussis, 1986; Lever and Weaver, 1986) characterized by

$$
H(p)=\mathrm{e}^{-T p},
$$

or

$$
h(t)=\delta(t-T) .
$$

The phase lag $\phi(\omega)=-\omega T$ is unbounded since it is proportional to the frequency. Price and Païdoussis (1986) propose $\beta=1 / a$ for the time-constant $T=\beta D / U$.

- with memory. The transfer function $H(p)$ is then a rational fraction and the phase lag is bounded. Granger and Paidoussis (1996) propose a first-order model and a two-order model. The first-order model is defined by the step response of the filter

$$
\Theta(t)=\left(1-\alpha_{1} \mathrm{e}^{-\delta_{1} a U / D t}\right) u(t)
$$

with $u(t)$ the Heaviside function. It illustrates the memory of the initial condition and is also referred as the Wagner function. The transfer function is

$$
H(p)=\mathcal{L}\left[\frac{d \Theta(t)}{d t}\right]=p \mathcal{L}[\Theta(t)]=1-\frac{\alpha_{1} p}{p+\delta_{1} a U / D} .
$$

By defining the two time-constants $T_{2}=\beta_{2} D / U$ and $T_{1}=\beta_{1} D / U$ where $\beta_{1}=1 / \delta_{1} a$ and $\beta_{2}=\beta_{1}\left(1-\alpha_{1}\right)$, the transfer function can be expressed on the following form:

$$
H(p)=\frac{1+T_{2} p}{1+T_{1} p}
$$

Fig. 4 shows this memory function for an in-line square array with a pitch ratio $P / D=1.5$. The constants $\alpha_{1}, \delta_{1}$ were obtained empirically by Granger and Paidoussis (1996) to fit with the critical velocity obtained by experiments. Meskell (2005) proposes a direct determination of the constants by modeling of the non-stationary wake by vortex method. The step response for the model of Price and Païdoussis (1986) is also displayed (Fig. 4) with the one obtained by the first-order model of Granger and Paidoussis (1996) without numerator $\left(T_{2}=0\right)$, as suggested by Meskell (2005). This model will be used thereafter for two reasons:

- The second order model does not induce great modification to the memory function for an in-line square array and gives critical velocities very close to those obtained by a first-order model.

- As $\alpha_{1}>1$ (Granger and Paidoussis, 1996), then $T_{2}<0$ in Eq. (7) and the step response presents a paradoxical behavior at the origin, since the force is negative before the change of sign. This phenomenon was not identified by the authors since it does not appear on the step response schematized in reference (Granger and Paidoussis, 1996). It is not very marked for an in-line square array because $T_{2} \ll T_{1}$, in contrast with a rotated pattern (Meskell, 2005). Without any other justification to accredit this paradoxical form, it will not be adopted here. A specific study of sensitivity to the zero of $H(p)$ is proposed below (cf. Section 4).

The first-order model of filtering chosen here is defined by its impulse response (with $T=T_{1}$ )

$$
h(t)=\frac{1}{T} \mathrm{e}^{-t / T},
$$

or its transfer function

$$
H(p)=\frac{1}{1+T p} .
$$



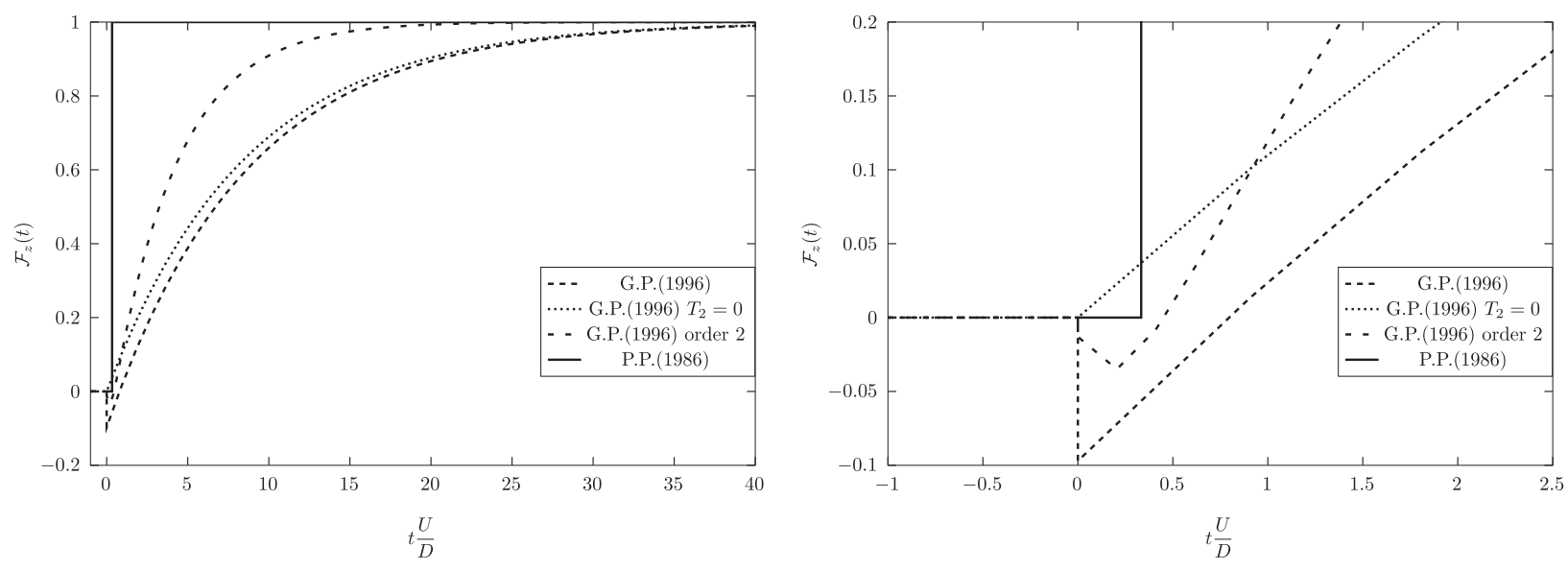

Fig. 4. Memory function for an in-line square array $P / D=1.5$.

Table 2

Parameters of the coupling model.

\begin{tabular}{|c|c|c|}
\hline Parameter & Notation & Value \\
\hline Drag coefficient (Price and Païdoussis, 1986) & $C_{D_{0}}$ & 2.3 \\
\hline Lift coefficient gradient (Price and Païdoussis, 1986) & $\left.\frac{\partial C_{L}}{\partial z^{\star}}\right|_{0}$ & 73 \\
\hline $\begin{array}{l}\text { Denominator reduced time-constant } \\
\text { (Granger and Paidoussis, 1996) }\end{array}$ & $\beta_{1}$ & 8.55 \\
\hline $\begin{array}{l}\text { Numerator reduced time-constant } \\
\text { (Granger and Paidoussis, 1996) }\end{array}$ & $\beta_{2}$ & 0.83 \\
\hline
\end{tabular}

Classically, the time constant of the first-order model is a function of the time-scale of advection $T_{A}=D / U$ with $\beta$ such that

$$
T=\beta \frac{D}{U}
$$

It is the form which gives the quasi-static model asymptotically $(H(p)=1)$ when $T p \rightarrow 0$, or in frequential terms when $\beta D \omega / U \rightarrow 0$, or $U_{r} \rightarrow \infty$ considering $\omega=\omega_{S}$ to obtain a global definition of the quasi-static assumption. An attempt to improve this time-constant model is proposed by Hémon (1999). The diffusion time-scale is certainly at least as important as because it is implied in the physique of separation. This remark justifies the need for considering the Reynolds number effects.

This low-pass first-order model has three parameters $C_{D_{0}}, \partial C_{L} /\left.\partial \bar{z}\right|_{0}, \beta$, that is to say the same parameters as for the timedelay model. The models with or without memory effects can thus be of the same level of complexity. The first two parameters are static parameters and are derived from the drag and lift coefficients at the equilibrium point. They depend on the pattern of the cylinder array and on the Reynolds number (Mahon and Meskell, 2012). The model of Lever and Weaver (1982) also includes static parameters but in greater number to describe the geometry of the stream tube. The third parameter is the dynamic one which fixes the time-constant of the mechanism of coupling. For the in-line square array with $P / D=1.5$, the parameter values were obtained from the literature as for Granger and Paidoussis (1996) and are given in Table 2. After analyzing the analogy of both models, we can compare them for two asymptotic situations.

\subsection{Phase-lag models for asymptotic values of Sc}

To compare the ability of the phase-lag models (bounded or unbounded) to estimate the critical velocity of instability, two asymptotic cases with respect to the Scruton number are considered. The stability criterion for high Scruton numbers of Price and Païdoussis (1986) is demonstrated by a different way and a new stability criterion for low Scruton numbers is proposed.

\subsubsection{Stability criterion for high Scruton numbers}

For high Scruton numbers, the reduced critical velocity is large. It can therefore be estimated considering $T p<1$ in the transfer function $H(p)$. So we have

$$
\frac{1}{1+T p} \approx 1-T p
$$


as it is the case for a memoryless model (Price and Païdoussis, 1986)

$$
\mathrm{e}^{-T p} \approx 1-T p
$$

Thus the approximation is the same for both models, whether it is for a time-delay model or a first-order low-pass filter model. The same approximation is valid for model in Eq. (7) since $T_{2}<T_{1}$ and

$$
\frac{1+T_{2} p}{1+T_{1} p} \approx 1-\left(T_{1}-T_{2}\right) p
$$

The functional diagram in Fig. 5 shows this asymptotic model for high Scruton number.

The stability condition is expressed by the relation $C_{S}+C_{G}>0$ with

$$
C_{G}=C_{D_{0}}-\left.\beta \frac{\partial C_{L}}{\partial Z^{\star}}\right|_{0}
$$

Formally, the expression (9) obtained by Price and Païdoussis (1986) is the same to the galloping instability criterion

$$
U_{r c}=\frac{4}{\left|C_{G}\right|} \mathrm{Sc}
$$

However, since this result is valid only for high Scruton numbers, this tube-array instability should not be confused with the galloping instability.

The time-delay model and the low-pass first-order model are equivalent to determine the critical velocity for high Scruton numbers. They show that the reduced critical velocity is proportional to the Scruton number. The paper of Granger and Paidoussis (1996) is in agreement with this result for a rotated pattern but not for an in-line square pattern with $U_{r c} \propto \sqrt{\mathrm{Sc}}$, which is not coherent with relation (9). It is however the behavior retained by Price (2001) or Païdoussis et al. (2010) in their synthesis book.

\subsubsection{Stability criterion for low Scruton numbers}

For low Scruton numbers, the reduced critical velocity is of the order of unity, but taking into account $\beta$ value, the critical velocity can be estimated with the approximation

$$
\frac{1}{1+T p} \approx \frac{1}{T p}
$$

that is sketched in the functional diagram of Fig. 6 .

The analysis of stability by the Nyquist criterion (Fung, 1955; Preumont, 2002) shows that the threshold of stability is reached when the magnitude of the open-loop Frequency-Response-Function equals one and phase-lag takes the value $180^{\circ}$. This is for the natural frequency of the structure $\omega_{S}$. That leads to

$$
U_{c}=\frac{C_{S}+C_{a 0}}{K_{a_{0}} M_{S}} \beta D K_{S}
$$

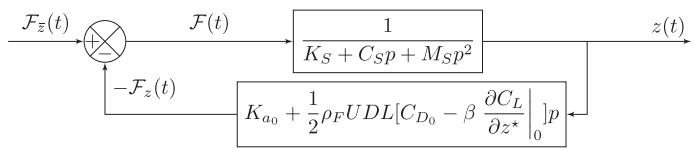

Fig. 5. Asymptotic model for high Scruton numbers.

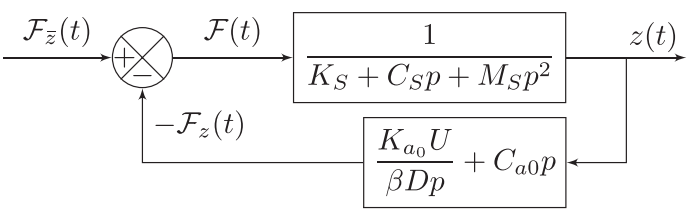

Fig. 6. Asymptotic model for low Scruton numbers. 
If the structural damping $\left(C_{S}\right)$ is negligible compared to the damping induced by the $\operatorname{drag}\left(C_{a 0}\right)$ or if like here $C_{S}=0$, the critical velocity is

$$
U_{c}=\sqrt{\frac{C_{D_{0}} \beta D}{\left.\frac{\partial C_{L}}{\partial z}\right|_{0} \omega_{n}}}
$$

The reduced critical velocity is

$$
U_{r c}=2 \pi \sqrt{\frac{C_{D_{0}} \beta}{\left.\frac{\partial C_{L}}{\partial z^{\star}}\right|_{0}},}
$$

or if the pitch velocity is used as reference

$$
U_{p r c}=2 \pi a \sqrt{\frac{C_{D_{0}} \beta}{\left.\frac{\partial C_{L}}{\partial Z^{\star}}\right|_{0}}}
$$

The critical velocity is asymptotically bounded when $\mathrm{Sc} \rightarrow 0$. It justifies the very weak influence of structural damping observed in practice on the critical velocity for low Scruton numbers (Price, 2001). This behavior is in perfect agreement with the formulation of the model, despite it is often regarded as paradoxical (Weaver, 2008). Confusion arises that it is not exactly, as generally reported, an instability controlled by damping but by the transient of the fluidelastic coupling.

To identify the validity domains for both asymptotic models, the Bode plot for all the one-time-constant models is given in Fig. 7. The cut-off reduced frequency $(-3 \mathrm{~dB},-45)$ for the first order model is $f_{r}^{-3 \mathrm{~dB}}=1 / 2 \pi \beta=0.0186$. If it is considered that the structure frequency is representative of the dominant oscillatory mode to define a global criterion, the validity of the asymptotic models is roughly conditioned by the two limits:

- $U_{r}>100$, then

$$
\frac{1}{1+T p} \approx \mathrm{e}^{-T p} \approx 1-T p
$$

In this domain, the time delay model and the low-pass first-order model are identical.

- $U_{r}<10$, then

$$
\frac{1}{1+T p} \approx \frac{1}{T p}
$$

In this domain, the time-delay model leads to very different results from the two other models, with switching stability/ instability when reduced velocity increases. The number of switches tends to infinity when the Scruton number tends to zero. Arbitrarily, Lever and Weaver (1986) recommend to consider only three stable domains.

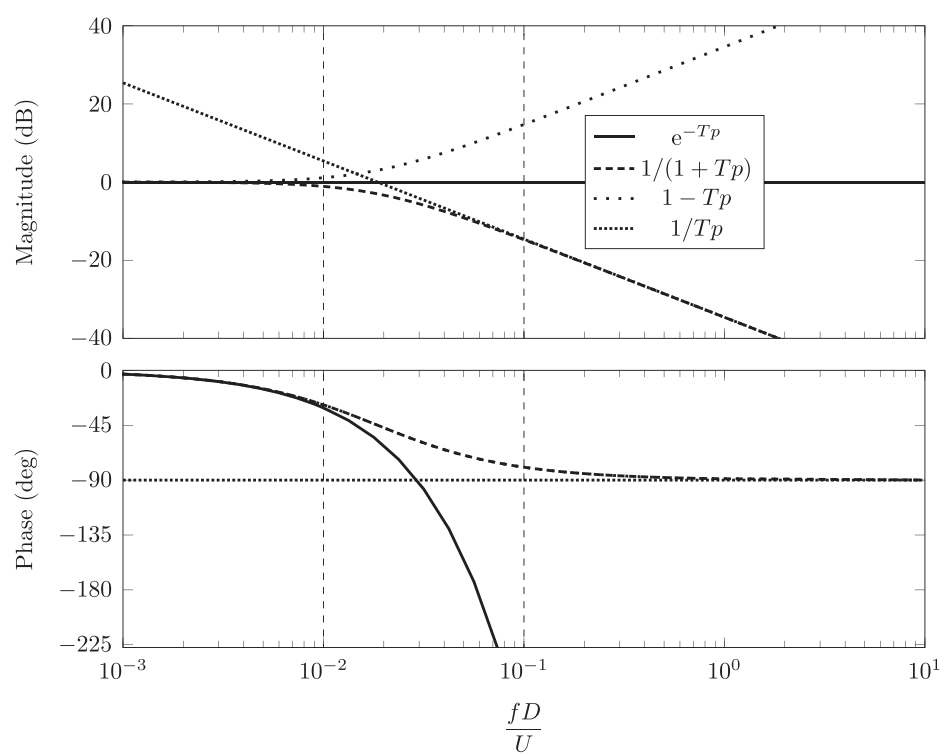

Fig. 7. Bode plot for one-time-constant models $(\beta=8.55)$. 


\section{Influence of the mass ratio}

To study the influence of the mass ratio, the lumped-parameter model is simulated with the software LMS Imagine.Lab AMEsim. Later, it will also allow:

- to refine the validity domain of the linear asymptotic models and to check their validity domains basing on frequency responses.

- to extend the analysis to higher-order models or nonlinear models,

- to develop a virtual prototype for the design of an experimental test bench.

Only the asymptotic case Sc $\rightarrow 0$ will be simulated here as it is known that, for high Scruton numbers, instability occurs because of antisymmetric stiffness and therefore the considered models are not suitable for. As the influence of the reduced velocity $U_{r}$ on the closed-loop modes will be analyzed by the root locus (i.e. the evolution of the poles in the complex plane), so we recall in Fig. 8 the characteristics of an oscillatory mode and of an aperiodic mode. $\omega_{n}$ is the natural frequency, $\omega_{p}$ the proper frequency and $\xi$ the reduced damping of the oscillatory mode. $T$ is the time constant of the aperiodic mode. A mode is faster when the pole goes to the left in the stable part of the complex plane. It is also pointed out (Preumont, 2002) that the $m$ open-loop poles are the starting points of the locii for $U_{r} \rightarrow 0$ and the $n$ zeros of the open-loop are the ending points for $U_{r} \rightarrow \infty$. When $m>n$ the root locii follow asymptotic directions in the complex plane.

In Fig. 9, the closed-loop poles are plotted for $0<U_{r}<40$ with a pitch of one. The open-loop poles $\left(U_{r}=0\right)$ are marked by crosses. The dashed lines corresponds to the stability threshold $(\xi=0)$ and to the iso- $\omega_{S}$ circle. The directions of the three locii when the reduced velocity increases are indicated by arrows. The aperiodic mode coming from the origin tends rapidly towards $-\infty$. The oscillatory mode coming from the mode of structure, becomes unstable with an asymptotic direction

(a)

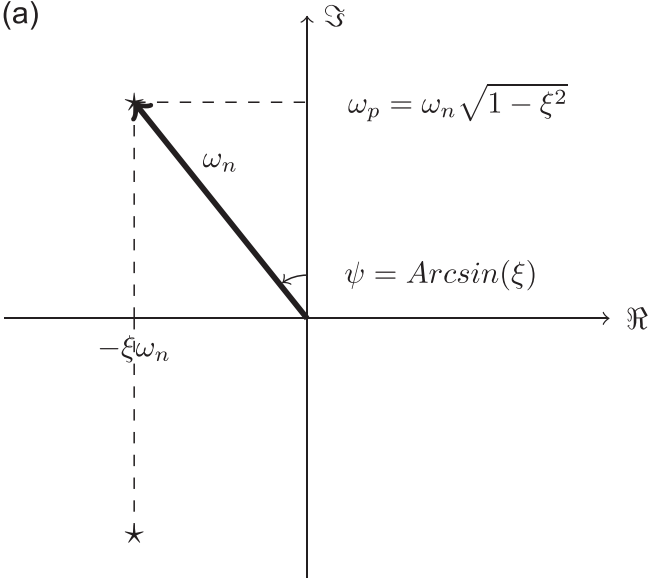

(b)

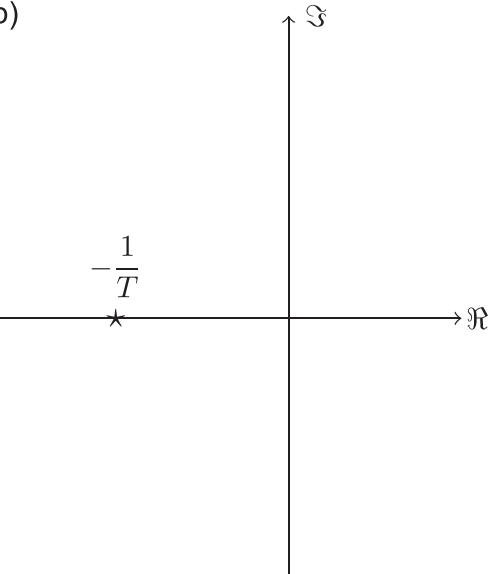

Fig. 8. Modal characteristics in the complex plane. (a) Oscillatory mode and (b) aperiodic mode.

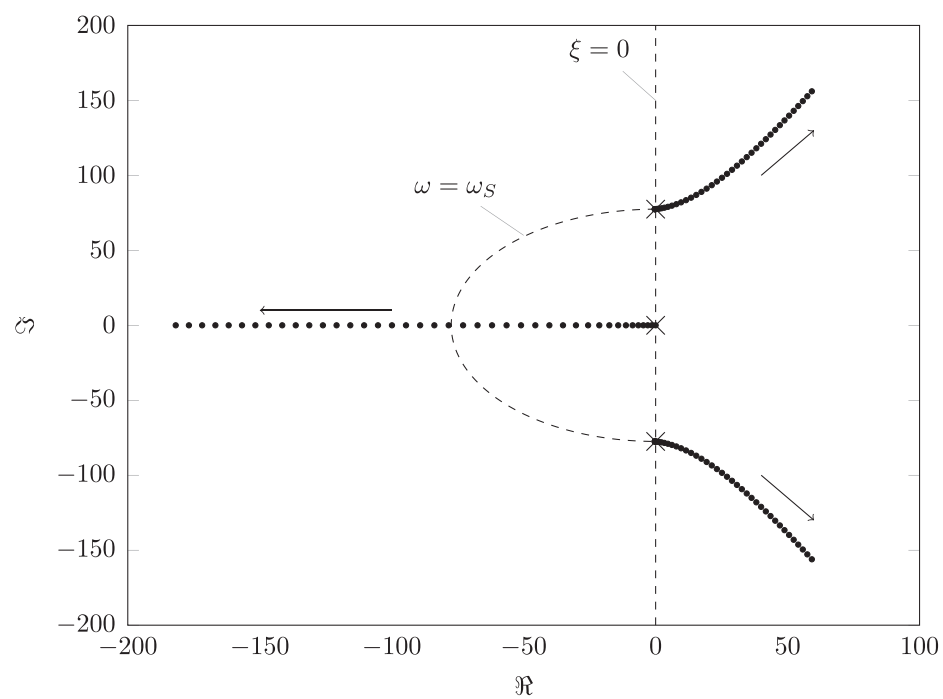

Fig. 9. Root locus with varying of reduced velocity $\left(\xi_{S}=0, m^{\star}=108\right)$. 
when $U_{r} \rightarrow \infty$. The critical reduced velocity $U_{r c}=3.26$ is exactly the value deduced from the asymptotic model of Eq. (12). Fig. 10 presents a zoom of Fig. 9 tracing the root locus for $0<U_{r}<4$ with a pitch of 0.2 . This allows us to analyze the loss of stability of the oscillatory mode depending whether the stabilizing effect of the drag is taken into account or not. When the reduced velocity increases, taking into account the drag $\left(C_{D_{0}}=2.3\right)$, the oscillatory mode begins to damp, otherwise $\left(C_{D_{0}}=0\right)$ the root locus directly goes to the unstable region. The drag damping plays a dominant role for low reduced velocity. It strongly influences the critical reduced velocity.

In order to analyze the influence of the mass ratio, the density of the fluid is increased up to $\rho=1000 \mathrm{~kg} / \mathrm{m}^{3}$. For the same structure, the mass ratio is $m^{\star}=0.13$ (water channel experiment) instead of $m^{\star}=108$ as before (wind tunnel experiment). It is pointed out that no modification of fluid viscosity is taken into account in the model. From the root locus for $0<U_{r}<4$ (Fig. 11) we can see that the damping of the oscillatory mode increases considerably and then decreases continuously. The maximum of the reduced damping $(\xi=0.86)$ is obtained for a reduced velocity $U_{r}=1.17$. The reduction in the natural frequency of the oscillatory mode is also much more important than in air. The inversion of trend of the frequency in the vicinity of the critical velocity results in a loop in the complex plane. We will see that this loop disappears if one takes the model of Granger and Paidoussis (1996). The critical reduced velocity $U_{r c}=3.85$ is slightly higher than $U_{r c}=3.26$, the value predicted by the asymptotic model (12). The global comparison is good considering the root locus for both models in Figs. 11 and 12. It appears that (i) $U_{r c}$ is not very sensitive to the mass ratio when $\xi_{S}=0$ and (ii) the integrator transfer function (10), is validated for low reduced velocities.

By neglecting the stabilizing effect of the drag $\left(C_{D_{0}}=0\right.$ ) the root locus (Fig. 13) exhibits a different topology for $0<U_{r}<4$. The influence of the drag is very important, especially when $m^{\star}$ is low. It is a result which can explain important differences between the in-line and rotated patterns because the geometry of the tube arrays is of great importance for the drag value.

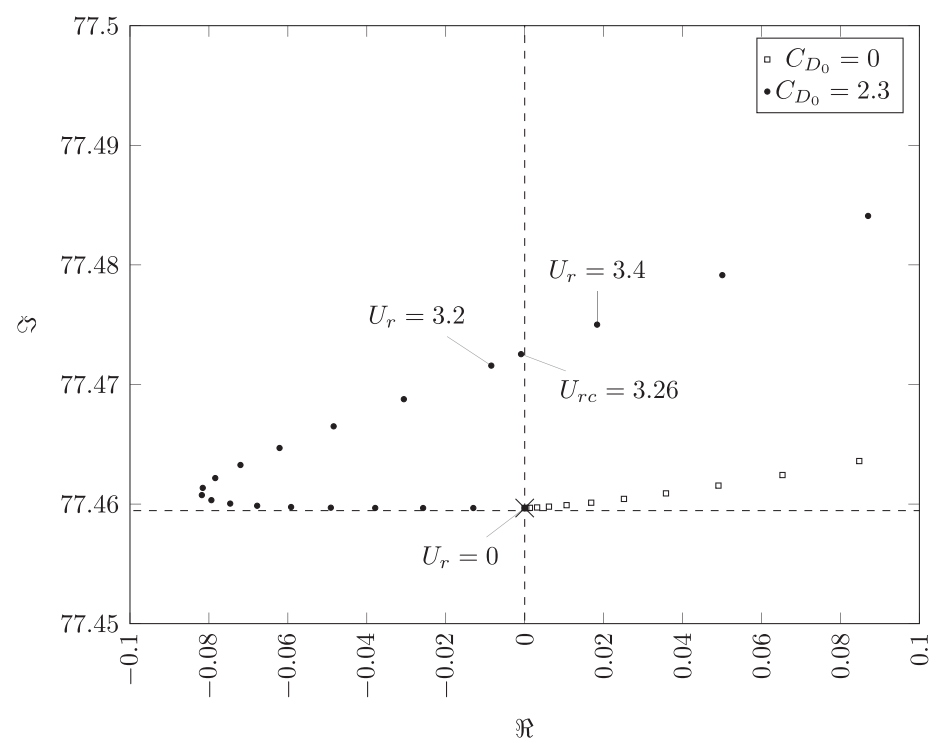

Fig. 10. Influence of the drag on the oscillatory mode $\left(\xi_{S}=0, m^{\star}=108\right)$.

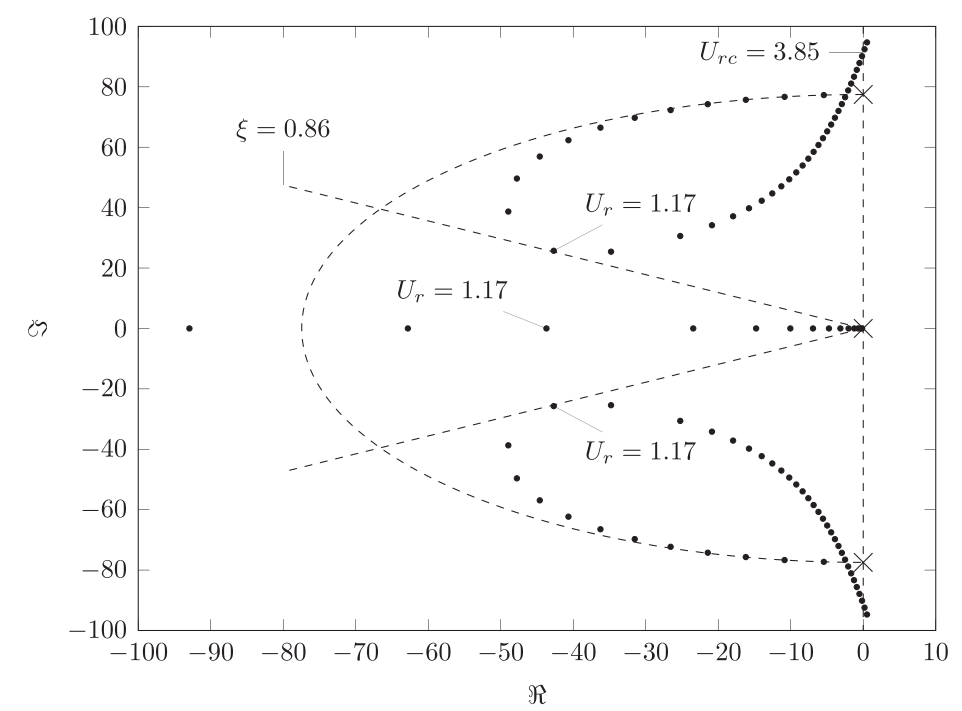

Fig. 11. Root locus with model (8) $\left(\xi_{S}=0, m^{\star}=0.13\right)$. 


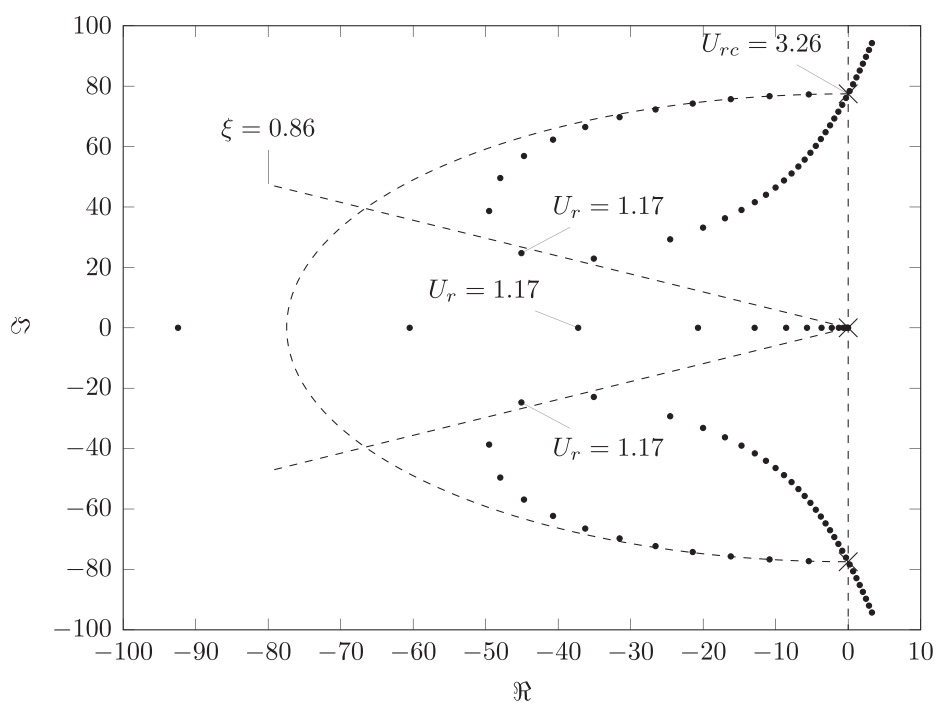

Fig. 12. Root locus with model (10) $\left(\xi_{S}=0, m^{\star}=0.13\right)$.

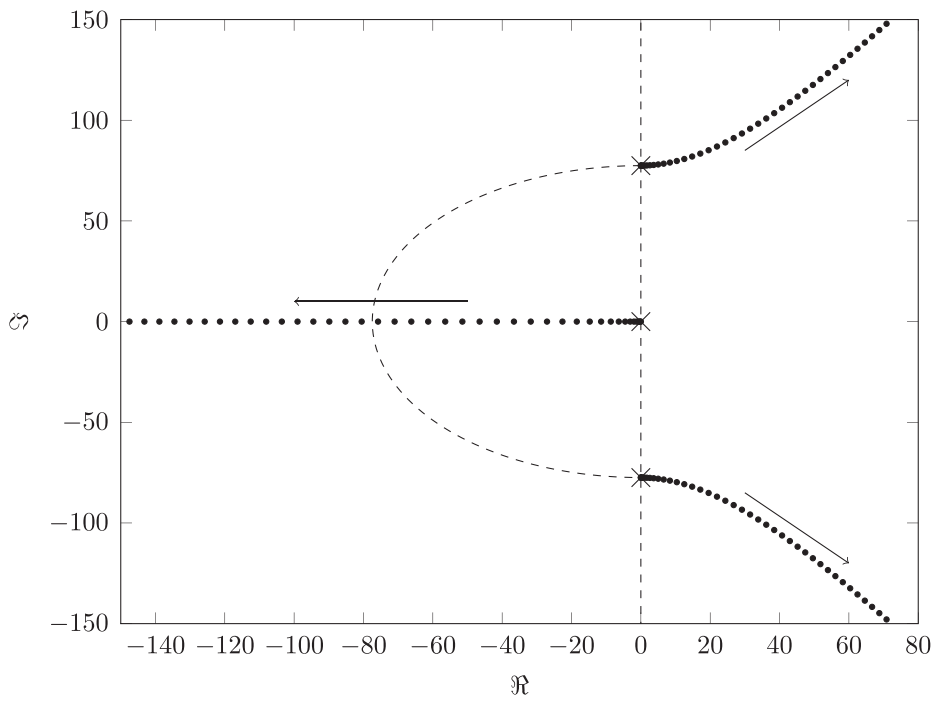

Fig. 13. Root locus $\left(\xi_{S}=0, m^{\star}=0.13, C_{D}=0\right)$.

With a structural damping such as $\mathrm{Sc}=0.1$, the root locus in Fig. 14 illustrates a behavior even more singular. To improve the representation, the step for the reduced velocity is not constant and the evolution of the fastest mode is not represented for $U_{r}>1.1$. Because of a junction of the poles of the oscillatory mode on the real axis, the system is characterized by three aperiodic modes for $1.088<U_{r}<1.093$. Beyond this value, the system has an aperiodic mode all the faster as $U_{r}$ increases and an oscillatory mode whose damping decreases and becomes zero for $U_{r c}=4$. In agreement with relation (11), this critical value is slightly higher than the value $U_{r c}=3.85$ obtained for $\xi_{S}=0$.

\section{Sensitivity to the zero of the transfer function}

In this section, we analyze the sensitivity of the first-order model to the magnitude and to the sign of the numerator time-constant $T_{2}$. We compare the results obtained with the transfer function (7) in the three cases $T_{2}=0, T_{2}>0$ and $T_{2}<0$. The last case is the choice of Granger and Paidoussis (1996). Although $\beta_{2}<\beta_{1}$ the zero of the transfer function plays a crucial role.

Considering $T_{2}=0$, for a high mass ratio $\left(m^{\star}=108\right)$, we showed that the natural frequency of the oscillatory mode increases continuously with the reduced velocity (Fig. 10), especially near the stability threshold. The increase is larger for $T_{2}>0$ as can be seen in Fig. 15. On the contrary, for $T_{2}<0$ there is a reduction in the frequency of the oscillatory mode during the crossing of the stability threshold. For higher reduced velocities (Fig. 16), the slope of the asymptote when $U_{r} \rightarrow \infty$ is sensitive to the numerator of the transfer function $H(p)$. For a low mass ratio (Fig. 17), this sensitivity is much more important. If the zero is positive $\left(T_{2}<0\right)$ the critical velocity is much lower $U_{r c}=1.15$ instead of $U_{r c}=3.85$ if $T_{2}=0$. If the zero is negative $\left(T_{2}>0\right)$ the asymptotic directions $\left(U_{r} \rightarrow \infty\right)$ is in the left half-plane; the closed-loop system is 


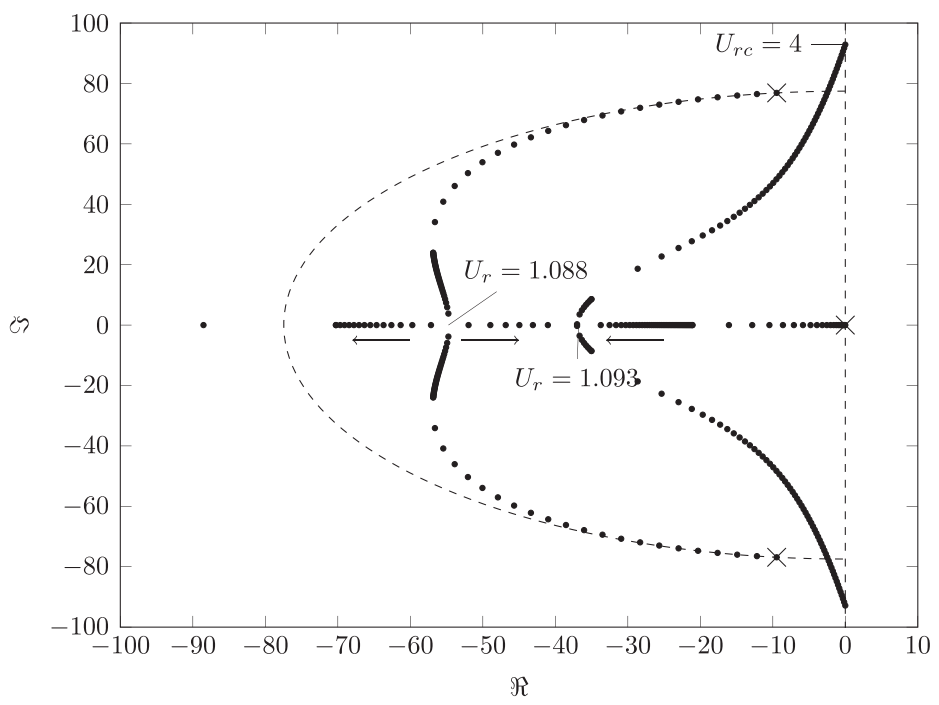

Fig. 14. Root locus $\left(m^{\star}=0.13, \mathrm{Sc}=0.1\right)$.

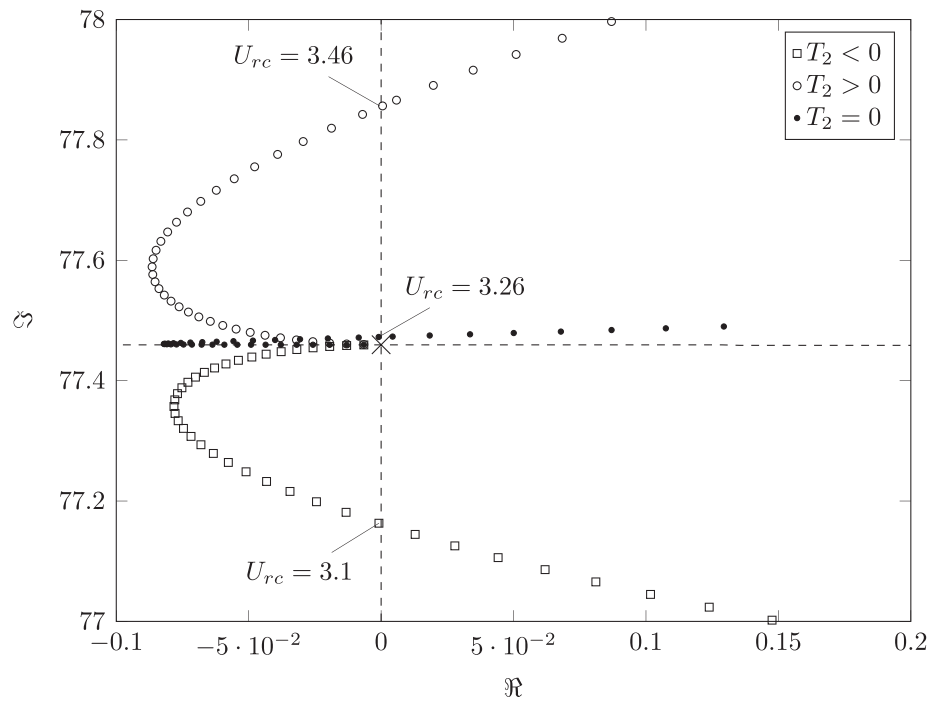

Fig. 15. Influence of $T_{2}$ on the oscillatory mode $\left(0<U_{r}<4, \xi_{S}=0, m^{\star}=108\right)$

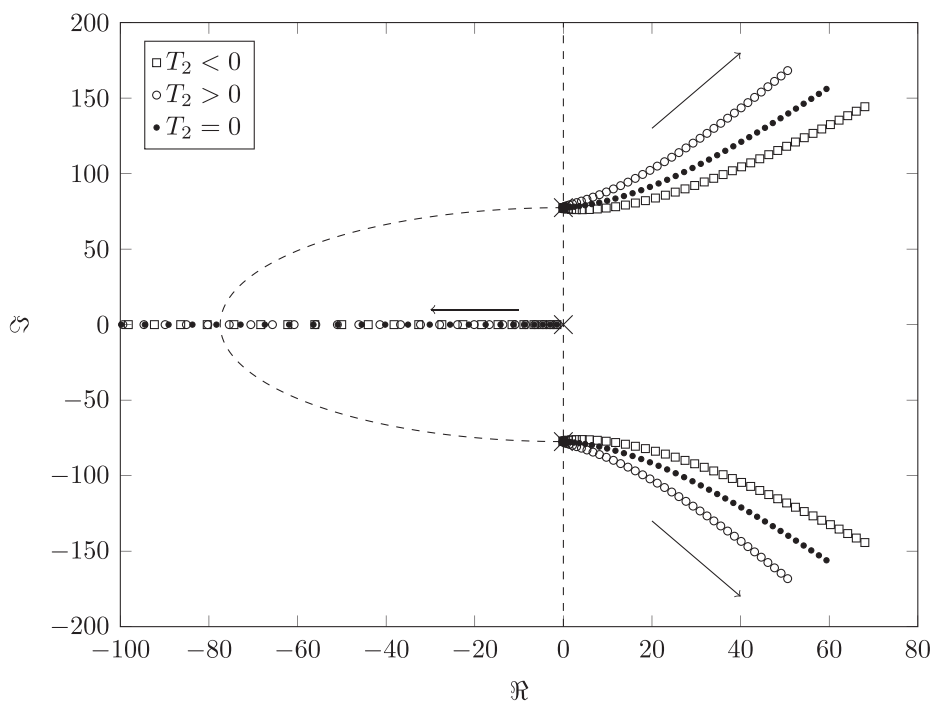

Fig. 16. Influence of $T_{2}$ on the oscillatory mode $\left(0<U_{r}<40, \xi_{S}=0, m^{\star}=108\right)$. 


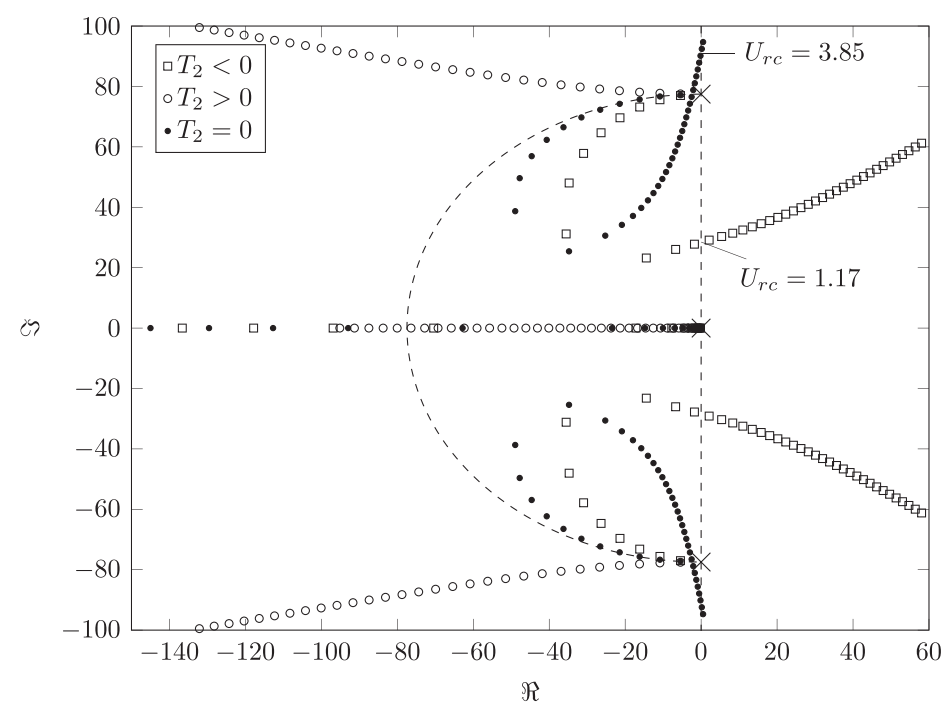

Fig. 17. Influence of $T_{2}$ on the oscillatory mode $\left(0<U_{r}<4, \xi_{S}=0, m^{\star}=0.13\right)$.

unconditionally stable. This influence on the form of the root locus is explained by the attraction effect of the open-loop zero since it is the ending-points of the root locus. When $U_{r} \rightarrow \infty$, the open-loop zero tends to $-\infty$ for $T_{2}>0$ or it tends to $+\infty$ for $T_{2}<0$. This attraction effect is used in control theory to inhibit an oscillatory mode with compensator zeros near system poles with notch-filter technique (Preumont, 2002).

It is thus noted that the paradoxical behavior of the step response of the filter, consequence of the negative sign of $T_{2}$, may be necessary to produce a dynamic instability. In contrast to the high-mass-ratio situation, the critical velocity is now very sensitive to the value of $\beta_{2}$. These results show a specificity of the tube bundle configuration, since the models classically used for airfoils and bluff bodies have always the same sign for pole and zero (Turbelin and Gibert, 2002). The paradoxical effect in the memory function, corroborated by the experimental results of Caillaud (1999), would thus have a legitimacy to model the dynamics of fluidelastic coupling in tube bundles.

\section{Discussion}

The analysis of fluidelastic instability in tube bundles of the results is often limited to the determination of a stiffness coefficient and a damping coefficient, to model the influence of the flow on the oscillatory mode of the structure. Considering a low-pass first-order transfer function to model the forces correlated with the movement, one can simply determine the expression of these coefficients as function of the time-constant $(T)$ of the model. For an harmonic movement of the structure $z(t)=z_{0} \sin (\omega t)$, the response of the fluidelastic model after the transient dies out is

$$
\left.\mathcal{F}_{z}(t)\right|_{t \rightarrow \infty}=-K_{a 0} z_{0}\left[\frac{1}{1+\omega^{2} T^{2}} z(t)-\frac{\omega T}{1+\omega^{2} T^{2}} \dot{z}(t)\right] .
$$

The term in phase with the displacement is the added stiffness and the term in phase with the velocity is the added damping. Preumont (1994) names it hereditary damping because it is the consequence of the history of the process. Both added coefficients depend on $\omega D / U$. The parameters of added-stiffness and added-damping are not constants but should be considered as functions of the reduced velocity, assuming $\omega \approx \omega_{S}$. In this class of models, one is not thus interested that in the branch of the root locus which crosses the imaginary axis (cf. for example Fig. 9), because only one oscillatory mode is taken into account. This modeling is valid only in the vicinity of the critical velocity where the oscillatory mode dominates. The mechanism of interaction is only considered through its consequences on one of the modes and the origin of instability is not described. In addition, it is assumed that the unstable mode comes necessarily from the branch of the root locus starting from the oscillatory mode of the structure. A single-time-constant model, derived from the first-order model by Granger and Paidoussis (1996), showed that it is not systematic when the mass number $m^{\star}$ is low. The elementary model does not take into account all the effects of the mass ratio: we omitted inertial effects in the quiescent fluid, which are generally taken into account by an added mass, in spite of the conceptual limitations of this approach exposed by Sarpkaya (2010). It does not bring into question the first conclusion:

For physical understanding, the fluidelastic model should not be restricted to the form of a memoryless process (without an own dynamic) since the instability is controlled by memory effects. The link of the added stiffness and added damping parameters with reduced velocity is only a trivial mean to take into account this memory. It is a better way to characterize the influence of the reduced velocity on the dynamics of the closed-loop system. 
A second element of discussion relates to the terminology often used to describe this kind of instability which may be misleading:

- The term single degree of freedom instability is misleading. If the structure in the quiescent fluid is an Single Degree Of Freedom oscillator, coupling with the flow introduces other dynamics which require increasing the model order. We considered here a closed-loop system with two degrees of freedom (the modal forms will be described by two variables of observation).

- The term damping-controlled suggests that a simple model of added-damping would be enough to describe the phenomenon correctly. If the reduced velocity is of the order of unity, the transients of the coupling process become important. In agreement with the experimental evidence, we showed that the critical velocity is then bounded when $\mathrm{Sc} \rightarrow 0$. It thus becomes practically insensitive to the structural damping. We also justified that the mass ratio is an important parameter, independently of reduced structural damping, since it dramatically modifies the topology of the root locus with significant added-stiffness effects.

To avoid confusion between instability in steam generators (low Scruton numbers) and the fully damping-controlled instabilities (galloping instability), the use of a specific terminology is essential. To name fluidelastic instabilities in two phase flow where the Scruton number is low (like in steam generators), one can suggest transient-controlled instability or instability by delayed stiffness.

In this study we compared the memoryless model of time delay with a memory model considering a time-constant only. Both models have the same level of complexity since both require only three parameters: $C_{D}, \partial C_{L} / \partial z^{\star}$, which are coefficients of static effort and $\beta$ which reflects the dynamics of the fluidelastic coupling. For large Scruton numbers, both models lead to the same critical velocity. They differ when Scruton number Sc $\rightarrow 0$. Asymptotically, the time delay model implies an infinity of stability/instability switches. In contrast, a rational fraction model implies that the reduced velocity is bounded, in better agreement with the experimental evidences.

For the first-order model with two time constants suggested by Granger and Paidoussis (1996), the influence of the zero in the coupling transfer function $\left(-1 / T_{2}\right)$ were analyzed. Contrary to airfoils or bluff bodies in an infinite medium, $T_{2}<0$ seems to be adapted to the tube bundles. The zero of the fluidelastic transfer function plays a crucial role on the route towards instability. Its influence is more important for small mass ratios $m^{\star}$; instability may disappear if the zero is negative. A rise in order of the fluidelastic model with other poles and zeros (real or complex) would make it possible to adapt the topology of the root locus and to include stability/instability switches. But, if this behavior was indeed observed (Chen and Jendrzejczyk, 1983), it was analyzed as a manifestation of non-linearities (Andjelic et al., 1992). The use of a memoryless model that is not compatible with the mechanism of diffusion is thus without significant interest.

\section{Conclusion}

Modeling of the fluidelastic forces for a single flexibly mounted cylinder in tube bundle is still an open subject to identify the linear form of the most suitable model. Despite of its simplicity, a first order model makes it possible to encompass a large variety of behavior, because of the complexity of the fluid-structure interaction when the mass ratio is low. A better identification of the fluidelastic linear model with respect to the geometric pattern and to the Reynolds number is necessary. The paradoxical behavior of the step response of the coupling filter proposed by Granger and Paidoussis (1996) seems relevant and would deserve a thorough study.

The asymptotic case of an undamped structure is a theoretical situation, but which allows to isolate and analyze the influence of the mass ratio. It can be studied by a test bench, controlling the structural damping and ensuring the similarity of geometry and Reynolds number with respect to applications. The numerical simulation with distributed parameters could be a complementary way for this identification work, in order to propose the proper model taking into account fluidelastic dynamics for tube bundle design (Longatte et al., 2013).

\section{Acknowledgments}

The present work has been carried out in the ANR BARESAFE project related to increasing the safety and reliability of nuclear power plant barriers. The author would like to acknowledge for their remarks and helpful discussions IMFT colleagues A. Sévrain, M. Braza, R. Bourguet, M. Elhimer and industrial partners E. Deri (EDF), E. Longatte, F. Baj (LaMSID).

\section{References}

Andjelic, M., Austermann, R., Popp, K., 1992. Multiple stability boundaries of tubes in a normal triangular cylinder array. Journal of Pressure Vessel Technology, Transactions of the ASME 114 (3), 336-343.

Axisa, F., 2001. Modélisation des systèmes mécaniques, Vibrations sous écoulements, vol. 4. Hermès science.

Blevins, R.D., 1994. Flow-Induced Vibration. Krieger.

Braza, M., Perrin, R., Hoarau, Y., 2006. Turbulence properties in the cylinder wake at high Reynolds numbers. Journal of Fluids and Structures 22 (6), $757-771$. 
Caillaud, S., 1999. Excitation forcée et contrôle actif pour la mesure des forces fluide-élastiques (Ph.D. thesis), Université de Paris VI.

Chen, S.S., 1987. A general theory for dynamic instability of tube arrays in crossflow. Journal of Fluids and Structures 1, 35-53.

Chen, S.S., Jendrzejczyk, J.A., 1983. Stability of tube arrays in crossflow. Nuclear Engineering and Design 75 (3), $351-373$.

Connors, H.J., 1970. Fluidelastic vibration of tube arrays excited by cross flow. In: Proceedings of the ASME Winter Annual Meet.

de Langre, E., 2006. Frequency lock-in is caused by coupled-mode flutter. Journal of Fluids and Structures 22 (6-7), 783-791.

Fung, Y.C., 1955. An Introduction to the Theory of Aeroelasticity. Dover Publications.

Gillen, S., Meskell, C., 2008. Variation of fluidelastic critical velocity with mass ratio and Reynolds number in a densely packed normal triangular tube array. In: Proceedings of Flow Induced Vibrations. Prague, Czech Republic.

Granger, S., Paidoussis, M.P., 1996. An improvement to the quasi-steady model with application to cross-flow-induced vibration of tube arrays. Journal of Fluid Mechanics 320, 163-184.

Hémon, P., 1999. An improvement of the time delayed quasi-steady model for the oscillations of circular cylinders in cross-flow. Journal of Fluids and Structures 13 (3), 291-307.

Hover, F.S., Techet, A.H., Triantafyllou, M.S., 1998. Forces on oscillating uniform and tapered cylinders in cross flow. Journal of Fluid Mechanics 363, 97-114.

Khalifa, A., Weaver, D., Ziada, S., 2012. A single flexible tube in a rigid array as a model for fluidelastic instability in tube bundles. Journal of Fluids and Structures 34, 14-32.

Lever, J.H., Weaver, D.S., 1982. A theoretical model for the fluidelastic instability in heat exchanger tube bundles. ASME Journal of Pressure Vessel Technology 104, 147-158.

Lever, J.H., Weaver, D.S., 1986. On the stability of heat exchanger tube bundles, part i: modified theoretical model. Journal of Sound and Vibration 107 (3), $375-392$.

Longatte, E., Baj, F., Hoarau, Y., Braza, M., Ruiz, D., Canteneur, C., 2013. Advanced numerical methods for uncertainty reduction when predicting heat exchanger dynamic stability limits: review and perspectives. Nuclear Engineering and Design 258 (0), $164-175$.

Mackowski, A.W., Williamson, C.H.K., 2011. Developing a cyber-physical fluid dynamics facility for fluid-structure interaction studies. Journal of Fluids and Structures 27, 748-757.

Mahon, J., Meskell, C., 2012. Surface pressure survey in a parallel triangular tube array. Journal of Fluids and Structures 34, $123-137$.

Meskell, C., 2005. On the underlying fluid mechanics responsible for damping controlled fluidelastic instability in tube arrays. In: ASME Conference Proceedings, pp. 573-581.

Païdoussis, M., Price, S., de Langre, E., 2010. Fluid-Structure Interactions: Cross-Flow-Induced Instabilities. Cambridge University Press.

Paidoussis, M.P., Price, S., Nakamura, T., Mark, B., Njuki Mureithi, W., 1989. Flow-induced vibrations and instabilities in a rotated-square cylinder array in cross-flow. Journal of Fluids and Structures 3 (3), 229-254.

Preumont, A., 1994. Random Vibration and Spectral Analysis, vol. 33. Kluwer Academic Publishers.

Preumont, A., 2002. Vibration Control of Active Structures, An Introduction. Kluwer Academic Publishers.

Price, S.J., Païdoussis, M.P., 1986. A single-flexible-cylinder analysis for the fluidelastic instability of an array of flexible cylinders in cross-flow. Journal of Fluids Engineering 108 (2), 193-199.

Price, S.J., 2001. An investigation on the use of Connors equation to predict fluidelastic instability in cylinder arrays. Journal of Pressure Vessel Technology $123,448-453$

Sarpkaya, T., 2010. Wave Forces on Offshore Structures. Cambridge University Press.

Silva, W., 2005. Identification of nonlinear aeroelastic systems based on the Volterra theory: progress and opportunities. Nonlinear Dynamics 39 (1), $25-62$.

Tanaka, H., Takahara, S., 1981. Fluid elastic vibration of tube array in cross flow. Journal of Sound and Vibration 77 (1), $19-37$.

Turbelin, G., Gibert, R.-J., 2002. Cfd calculations of indicial lift responses for bluff bodies. Wind and Structures An International Journal 5 (2), 245-256.

Wagner, H., 1925. Über die entstehung des dynamischen auftriebes von tragflügeln. ZAMM-Journal of Applied Mathematics and Mechanics/Zeitschrift für Angewandte Mathematik und Mechanik 5 (1), 17-35.

Weaver, D.S., 2008. Some thoughts on the elusive mechanism of fluidelastic instability in heat exchanger tube arrays. In: Proceedings of Flow Induced Vibrations. Prague, Czech Republic. 